\title{
Bilateral macular infarction as an ocular manifestation of systemic lupus erythematosus (SLE)
}

\author{
This article was published in the following Dove Press journal: \\ Clinical Ophthalmology \\ 12 September 2014 \\ Number of times this article has been viewed
}

\section{Chih-Ling $\mathrm{Hu}$ \\ Kai-Ling Peng}

Department of Ophthalmology, Chi Mei Medical Center, Tainan, Taiwan, Republic of China
Correspondence: Kai-Ling Peng Department of Ophthalmology, Chi Mei Medical Center, 90I Zhonghua Road,

Yongkang District, Tainan, Taiwan 7I0, Republic of China

Tel +886628I 28II ext 57247

Email caropk165@gmail.com

\begin{abstract}
We report a rare case of bilateral macular infarction as an ocular presenting sign of systemic lupus erythematosus (SLE). A 29-year-old woman presented to our ophthalmologic clinic with a 1-week history of progressive visual loss in her left eye after she had visited a rheumatologic clinic where SLE was diagnosed. At examination, best-corrected visual acuity (BCVA) of the right eye was $6 / 6$, and for the left was counting fingers. Fundus examination revealed perivascular hard exudates along some branches of vessels in both eyes. After pulse therapy, her BCVA in the right eye declined to $6 / 30$ and in the left improved to $3 / 60$. She was administered sub-Tenon's injections of triamcinolone acetonide $50 \mathrm{mg} /$ week in both eyes for 3 weeks. Her BCVA improved to 3/6 in her right eye and remained at 3/60 in her left eye. Macular infarction is an uncommon but most severe complication of SLE. Early and regular exam of the fundus in patients with SLE is necessary to avoid progression of severe ocular complications.

Keywords: bilateral macular infarction, systemic lupus erythematosus
\end{abstract}

\section{Introduction}

Systemic lupus erythematosus (SLE) is an autoimmune inflammatory disease affecting multiple systems by circulating autoantibodies and the destruction of the accumulating immune complex. Visual system involvement has been found in up to a third of patients with SLE. ${ }^{1}$ In the majority of cases, visual prognosis is excellent. However, vaso-occlusive retinopathy, even inducing macular ischemia and infarction, a rare and severe ocular complication of SLE, can result in poor visual outcome.

Initial treatment for vaso-occlusive retinopathy is usually high-dose systemic corticosteroids. During the treatment period, such visual symptoms as deterioration of vision and visual field defect are indicators of progression of SLE retinopathy. Sub-Tenon's injection of corticosteroids, steroid-sparing immunosuppressive agents, anticoagulation agents, and panretinal photocoagulation are applied in more severe cases or worse conditions.

We report a rare case of bilateral macular infarction occurring in a patient who was simultaneously diagnosed with SLE. Under treatment with pulse therapy and intravenous steroids, her vision deteriorated progressively. When this was combined with an adjuvant therapy of multiple shots of posterior sub-Tenon's injection of triamcinolone acetonide (TA), the vision in one eye unexpectedly improved and was maintained at follow-up.

\section{Case report}

The 29-year-old female patient suffered from loss of vision in the left eye for 1 week. She came to our ophthalmologic clinic, where best-corrected visual acuity (BCVA) of the right eye was $6 / 6$, and the left was counting fingers at $20 \mathrm{~cm}$. According to the 
patient, she had been to the rheumatologic clinic, where SLE was diagnosed according to the symptoms of arthralgia over the bilateral fingers and knees for 2-3 months and fever on and off for 1 month. Laboratory testing revealed anemia with a low hemoglobin level of $8.0 \mathrm{~g} / \mathrm{dL}$, proteinuria, an antinuclear antibody titer of 1:2,560, anti-double-stranded deoxyribonucleic acid (dsDNA) titer of $>400 \mathrm{IU} / \mathrm{mL}$, and an anticardiolipin antibody titer in normal range. Therefore, the diagnosis of SLE was confirmed, and she was treated with oral steroids for a week. In our clinic, the results of the external and anterior segments were unremarkable. Posterior segment examination showed white perivascular exudate in her right eye and multiple patches of cotton-wool spots around the disk and macula in her left eye (Figure 1).

She was admitted to the rheumatology ward for further evaluation and treatment. However, BCVA of the right eye declined to 6/10. Fundus examination showed new multiple soft exudates in the macula, with a small branch of sheathing vessels and perivascular hard exudate along multiple branches in her right eye. Due to progressively worse vision in the right eye, intravenous steroids with methylprednisolone $1,000 \mathrm{mg} /$ day for 3 days were given and then $80 \mathrm{mg} /$ day for 10 days thereafter. Her anti-dsDNA titer regressed from $>400$ to $90 \mathrm{IU} / \mathrm{mL}$. Arthralgia of the bilateral fingers and knees was resolved. Unfortunately, her right eye vision went down to $6 / 30$ on the 4 th day at a dose of $80 \mathrm{mg}$ intravenously, with less perivascular exudate but increasing cotton-wool spots and multiple arterioles narrowing at the macula in her right eye, while her left eye improved to 3/60, with confluent macular cotton-wool spots presenting like cherry-red spots and attenuated smaller arterioles (Figure 2A). Fluorescein angiography (FA) showed an extensive macular nonperfusion area in the left eye (Figure 2B) and a small branch of a capillary nonperfusion zone at the macula in the early phase, with perivascular leakage of multiple arterioles at the late phase in the right eye (Figure 2C). Ocular coherence tomography (OCT) showed localized retina edema with subfoveal fluid in both eyes.

She underwent posterior sub-Tenon's injections of TA $50 \mathrm{mg}$ per week for 3 weeks in both eyes (Figure 3). Simultaneously, she was given oral antiplatelet medication of pentoxifylline $250 \mathrm{mg}$ twice a day and steroids tapering to $40 \mathrm{mg} /$ day 2 weeks later. Her anti-dsDNA titer regressed from 90 to $45 \mathrm{IU} / \mathrm{mL}$, while her right eye vision gradually improved to $3 / 6$ and the left eye remained at 3/60 after a month of first posterior sub-Tenon's injections. There was obviously decreasing macular soft exudates in both eyes (Figure 4A), with less perivascular leakage in each eye and a smaller capillary nonperfusion area in the right eye from FA (Figure 4B). OCT showed no more subfoveal fluid and fovea edema in either eye, with macular thinning in the left eye.

She kept following up at our clinic, with the dose of oral steroids tapering according to the manifestations of her bilateral posterior segments. BCVA of the right eye upgraded to $6 / 7.5$, and the left eye remained at 3/60 after 6 weeks of first posterior sub-Tenon's injections. Her bilateral vision was maintained for 4 months. There were no cotton-wool spots over the posterior pole in either eye (Figure 5A), with a smaller nonperfusion area in the right eye (Figure 5B).

\section{Discussion}

Retinal disease affects around $10 \%$ of patients with SLE. Mild lupus retinopathy showed cotton-wool spots, perivascular hard exudates, retinal hemorrhages and vascular

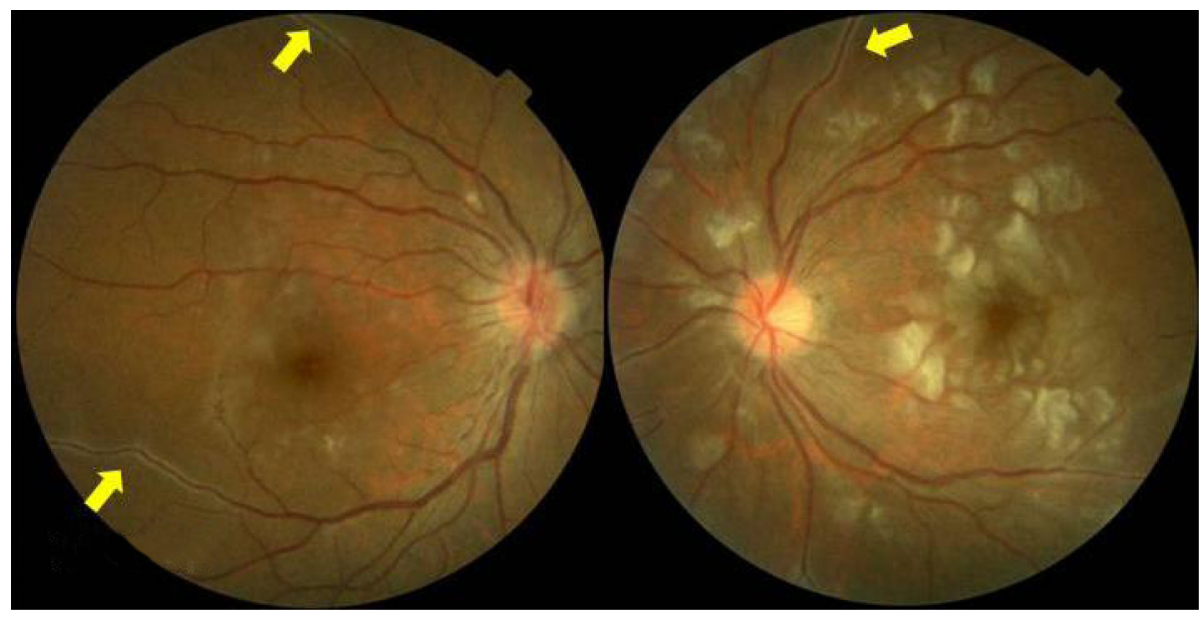

Figure I There was white perivascular hard exudate (yellow arrow) along the vessels in both eyes, and multiple patches of cotton-wool spots around the disk and macula in her left eye. 


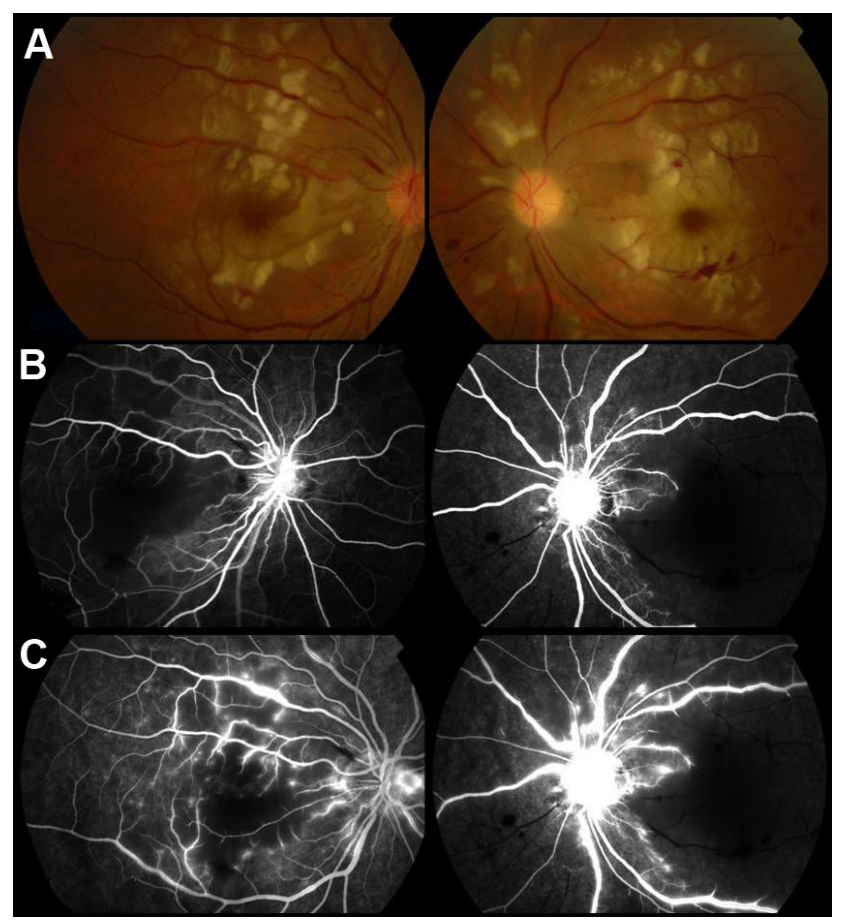

Figure 2 (A) After pulse therapy, there were increasing cotton-wool spots and multiple arterioles narrowing at the macula in her right eye and confluent macular cotton-wool spots presenting like cherry-red spots with attenuated smaller arterioles in her left eye. (B) At the early phase in the macula, a small branch of a capillary nonperfusion zone was observed in the right eye, and multiple branches of arterioles were occluded in the left eye. (C) At the late phase in the macula, perivascular leakage of multiple arterioles was observed in both eyes. There was an extensive capillary nonperfusion zone in the left macula.

tortuosity. ${ }^{2}$ Moderately severe cases may have focal or generalized arteriolar constriction and venous tortuosity. In severe groups, there is occlusion of retinal arterioles and consequent retinal infarction, vaso-occlusive retinopathy, or retinal vasculitis. ${ }^{3,4}$ This means the progression of SLE retinopathy is at first from perivascular hard exudates, then arteriole constriction and finally arteriole occlusion.

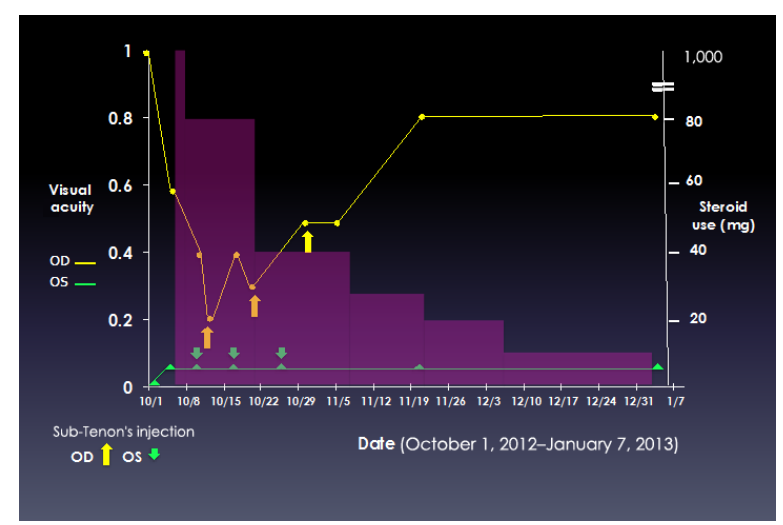

Figure 3 The relationship of visual acuity and steroid use under sub-tenon injection and intravenous way.

Abbreviations: OD, oculus dexter; OS, oculus sinister

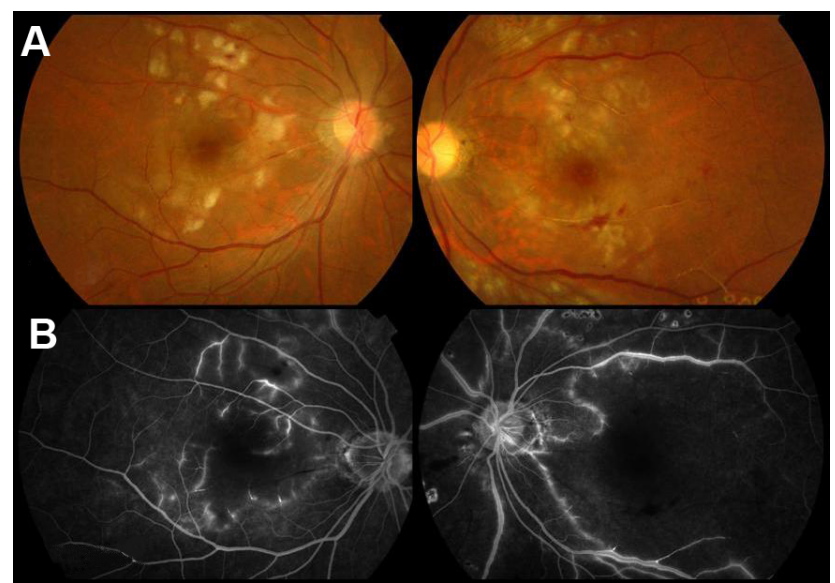

Figure 4 (A) After sub-Tenon's injection of triamcinolone acetonide $50 \mathrm{mg} /$ week in both eyes for 3 weeks, there were obviously decreasing soft exudates in the macula in both eyes. (B) At the late phase in the macula, there was less perivascular leakage in each eye and a smaller capillary nonperfusion area in the right eye.

Microscopically, autoantibodies attacking the walls of arterioles make vascular permeability increase, with presentations of perivascular exudates along vessels and severe vascular leakage on FA. Immune-complex deposition in the arterioles allows intravascular space narrowing. The perivascular neural cells become ischemic, with manifestations of more cotton-wool spots. ${ }^{5,6} \mathrm{FA}$ shows the nonperfusion area. As the arterioles around the macula totally occlude with sheathing vessels, the vision deteriorates irreversibly. Shein et al concluded after reviewing the literature that the visual prognosis of macular ischemia or infarction presenting as the initial sign of SLE with no evidence of elevated anticardiolipin antibody titers tends to be poor, despite treatment with high-dose systemic corticosteroids and noncorticosteroid immunosuppressive agents. ${ }^{7}$

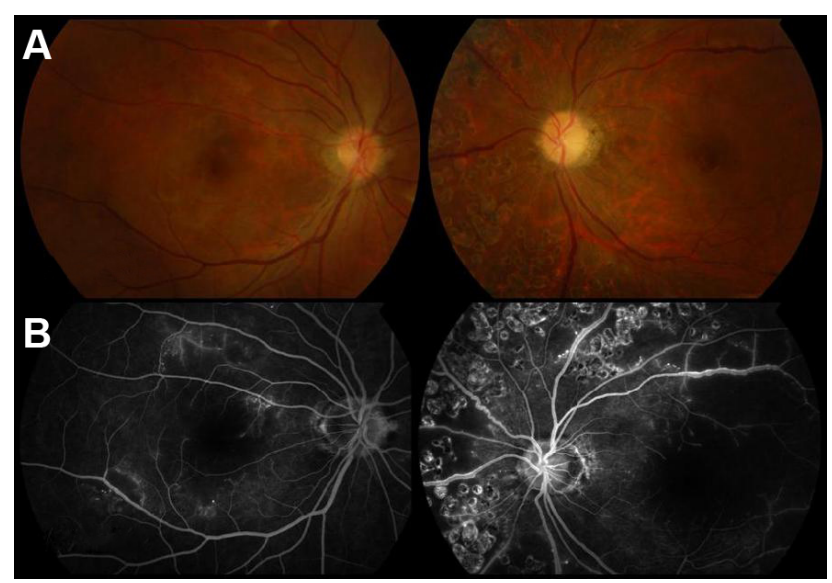

Figure 5 (A) There were no cotton-wool spots over posterior pole in either eye. The left disk seemed mildly waxy pale. (B) At the late phase in the macula, there was a smaller nonperfusion area in the right eye. 
From our case, the perivascular hard exudation of both eyes was partially resolved, with anti-dsDNA decreasing after pulse therapy. FA revealed continued vascular leakage at the late phase representing high vascular permeability induced by vascular continuous inflammation. However, the cotton-wool spots dispersed over the macular area increased rapidly with worse vision. We supposed that perivascular exudates seemed to be resolved at initial periods by high-dose intravenous steroids through arterioles without occlusion. Accumulation of a large amount of autoantibodies or immune complexes makes intravascular space narrow or totally occluded. Thereafter, the concentration of intravenous steroids could be too low to wash out excessive autoantibodies or the immune complex. The area of the perivascular neural cells short of nutrition and oxygen gradually expands and advances.

Increasing steroid levels over the posterior pole to eliminate excessive autoantibodies or immune complex in the vessels should be achieved. Posterior sub-Tenon's injection of TA, a long-acting corticosteroid, might be a good adjuvant treatment. Delivery of TA from the posterior subTenon's space to the extracellular choroidal matrix through transscleral diffusion, demonstrated by Kovacs et al could boost the localized concentration of steroids lasting at least 1 month. ${ }^{8}$ We assume that increasing steroid density over posterior segments could not only deplete more autoantibodies or immune complexes to widen the narrowing vessels with reversing the condition of ischemia but also relieve the edema of perivascular neural cells and extracellular tissue induced by inflammation simultaneously. For totally occluded vessels with infarction, the situation would be irreversible. With regard to our case, cotton-wool spots over the posterior pole of bilateral eyes became obviously diminished after injections in both eyes. FA showed less vascular leakage in both eyes, with a smaller area of capillary dropout in the right eye. OCT showed resolved subfoveal fluid in both eyes. The vision in the right eye improved, but the left eye remained the same.
As the systemic symptoms of SLE resolved quickly with significant reducing of anti-dsDNA, systemic corticosteroids were considered to taper gradually. However, retinal signs and vision in our case improved gradually; not as fast as the systemic ones. Tapering systemic corticosteroids should be controlled carefully according to the retinal presentations and the visual change, which often parallel the severity of systemic inflammation and may indicate inadequate control of the systemic disease. ${ }^{9}$ In conclusion, posterior sub-Tenon's injection of TA is an adjuvant treatment for refractory macular infarction, an uncommon but sight-threatening ocular complication, even if vision is worse after the use of highdose systemic corticosteroids.

\section{Disclosure}

The authors report no conflicts of interest in this work.

\section{References}

1. Read RW. Clinical mini-review systemic erythematosus and the eye. Ocul Immunol Inflamm. 2004;12:87-99.

2. Coppeto J, Lessel S. Retinopathy in systemic lupus erythematosus. Arch Ophthalmol. 1977;95:794-797.

3. Jabs DA, Fine SL, Hochberg MC, Newman SA, Heiner GG, Stevens MB. Severe retinal vaso-occlusive disease in systemic lupus erythematosus. Arch Ophthalmol. 1986;104:558-563.

4. Hall S, Buettner H, Luthra HS. Occlusive retinal vascular disease in systemic lupus erythematosus. J Rheumatol. 1984;11:846-850.

5. Nag TC, Wadhwa S. Histopathological changes in the eyes in systemic lupus erythematosus: an electron microscope and immunohistochemical study. Histol Histopathol. 2005;20:373-382.

6. Nag TC, Wadhwa S. Vascular changes of the retina and choroid in systemic lupus erythematosus: pathology and pathogenesis. Curr Neurovasc Res. 2006;3:159-168.

7. Shein J, Shukla D, Reddy S, Yannuzzi LA, Cunningham ET. Macular infarction as a presenting sign of systemic lupus erythematosus. Retin Cases Brief Rep. 2008;2:55-60.

8. Kovacs K, Wagley S, Quirk MT, et al. Pharmacokinetic study of vitreous and serum concentrations of triamcinolone acetonide after posterior subTenon's injection. Am J Ophthalmol. 2012;153:939-948.

9. Areval JF, Lowder CY, Muci-Mendoza R. Ocular manifestations of systemic lupus erythematosus. Curr Opin Ophhthalmol. 2002; 13:404-410.
Clinical Ophthalmology

\section{Publish your work in this journal}

Clinical Ophthalmology is an international, peer-reviewed journal covering all subspecialties within ophthalmology. Key topics include: Optometry; Visual science; Pharmacology and drug therapy in eye diseases; Basic Sciences; Primary and Secondary eye care; Patient Safety and Quality of Care Improvements. This journal is indexed on Submit your manuscript here: http://www.dovepress.com/clinical-ophthalmology-journal

\section{Dovepress}

PubMed Central and CAS, and is the official journal of The Society of Clinical Ophthalmology (SCO). The manuscript management system is completely online and includes a very quick and fair peer-review system, which is all easy to use. Visit http://www.dovepress.com/ testimonials.php to read real quotes from published authors. 